SLAC-PUB-11268

June 2005

\title{
A PROPOSAL FOR A NEW HOM ABSORBER IN A STRAIGHT SECTION OF THE PEP-II LOW ENERGY RING*
}

S. Weathersby, M. Kosovsky, N. Kurita, A. Novokhatski, J. Seeman

Stanford Linear Accelerator Center, Stanford University, Stanford, CA 94309

\begin{abstract}
Attainment of high luminosity in storage ring colliders necessitates increasing stored currents and reducing bunch lengths. Consequently, intense beam fields will scatter more power into higher order modes from beam line sources such as collimators, masks and tapers. This power penetrates into sensitive components such as a bellows, causing undesirable heating and limits machine performance. To overcome this limitation we propose incorporating ceramic absorbers in the vicinity of the bellows to damp beam induced modes while preserving a matched impedance to the beam. This is accomplished with an absorber configuration which damps TE dipole and quadrupole traveling waves while preserving TM monopole propagation. A scattering parameter analysis is presented utilizing properties of commercial grade ceramics and indicates a feasible solution.
\end{abstract}

Contributed to Particle Accelerator Conference

Knoxville, Tennessee, USA

May 16 - May 20, 2005

*Work supported by Department of Energy contract DE-AC02-76SF00515. 


\title{
A PROPOSAL FOR A NEW HOM ABSORBER IN A STRAIGHT SECTION OF THE PEP-II LOW ENERGY RING*
}

\author{
S. Weathersby, M. Kosovsky, N. Kurita, A. Novokhatski, J. Seeman, Stanford Linear Accelerator Center, USA \\ Abstract \\ Attainment of high luminosity in storage ring collid- \\ ers necessitates increasing stored currents and reducing \\ bunch lengths. Consequently, intense beam fields will \\ scatter more power into higher order modes from beam \\ line sources such as collimators, masks and tapers. This \\ power penetrates into sensitive components such as a bel- \\ damp HOMs while preserving beam stability it is desirable \\ to tailor the absorbing structure to have a transparent beam \\ impedance. We present a design for a bellows structure \\ in a LER straight section incorporating water cooled ce- \\ ramic absorbers in a geometry which damps HOMs pro- \\ duced from a nearby fixed horizontal and vertical collima- \\ tor while minimizing beam impedance.
} lows, causing undesirable heating and limits machine performance. To overcome this limitation we propose incorporating ceramic absorbers in the vicinity of the bellows to damp beam induced modes while preserving a matched impedance to the beam. This is accomplished with an absorber configuration which damps TE dipole and quadrupole traveling waves while preserving TM monopole propagation. A scattering parameter analysis is presented utilizing properties of commercial grade ceramics and indicates a feasible solution.

\section{INTRODUCTION}

The PEP-II B-factory storage ring collides $3 \mathrm{GeV}$ positrons with $10 \mathrm{GeV}$ electrons in the BaBar detector IP region. Run 4 achieved production luminosities of $9 \times 10^{33}$ $\mathrm{cm}^{-2} \mathrm{~s}^{-1}$ at currents of $2.4 \mathrm{~A}$ positrons in the low energy ring (LER) and $1.5 \mathrm{~A}$ electrons in the high energy ring (HER) in the form of bunch trains comprising typically 1600-1700 $1.3 \mathrm{~cm}$ long bunches. At these currents and bunch lengths various beam line components experience anomalous heating attributed to higher order beam induced electromagnetic fields (HOMs). These fields propagate from nonuniformities in the beam chamber cross sections which act as scattering centers for the beam fields.

Collimators along a LER straight section were demonstated to generate such fields. Nearby bellows see the effect of beam proximity to the collimator. For a vertical collimator in a LER straight section HOM heating is shown to depend strongly on the beam vertical offset from the collimator[1]. HOM propagation was found to travel along the beam direction and to have a quadratic dependence on the bunch length.

The requirement for higher luminosity entails higher ring currents at the 3-4 A levels as well as shorter bunch lengths of $0.8 \mathrm{~cm}$, both of which will contribute to HOM heating. HOM ceramic absorbers have been placed on the walls of beam chambers near potential HOM generators, such as crotches, collimators, and synchrotron radiation masks[2]. While this may be effective in damping HOMs this also can affect the beam and induce instability by presenting additional beam impedance. To properly

\footnotetext{
* Work supported by the U.S. Department of Energy under contract number DE-AC03-76SF00515.
}

\section{COLLIMATOR HIGHER ORDER MODES}

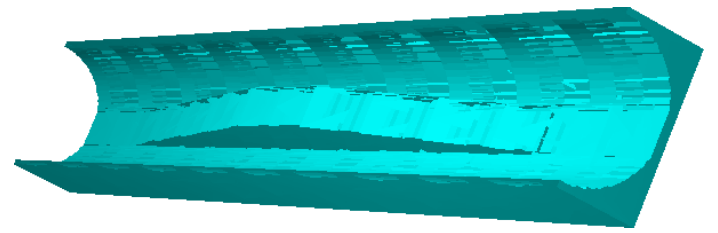

Figure 1: LER straight collimator

\section{Loss Factors}
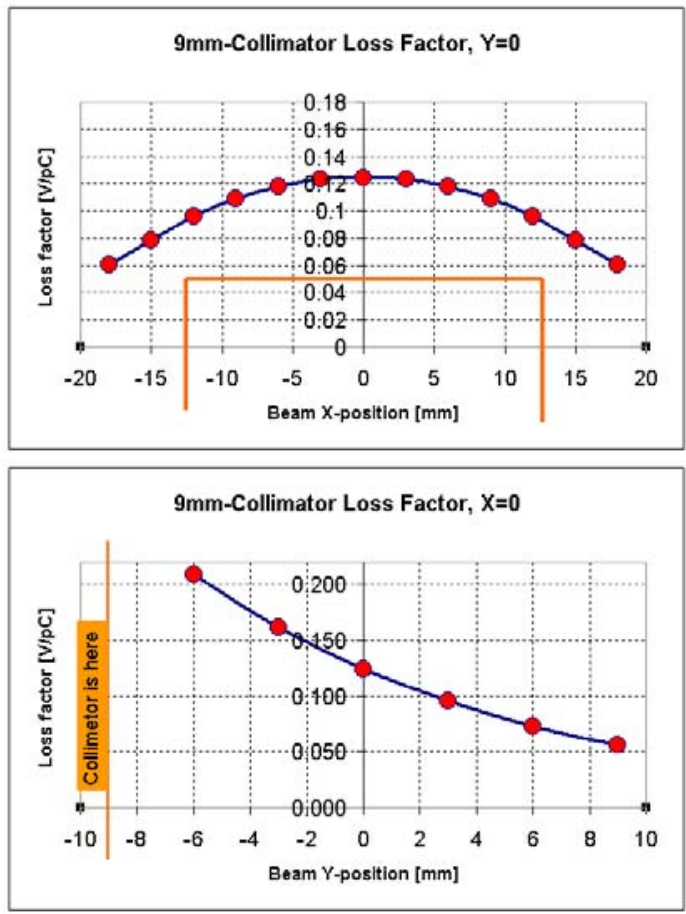

Figure 2: Loss factor as a function of beam position

To understand the HOM generation at the collimator of a LER straight section we calculate loss factors as function of beam position relative to the collimator and as a function 


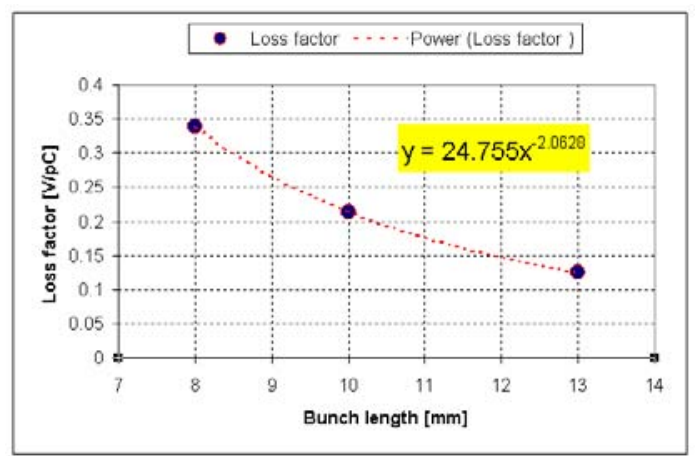

Figure 3: Loss factor bunch length dependence

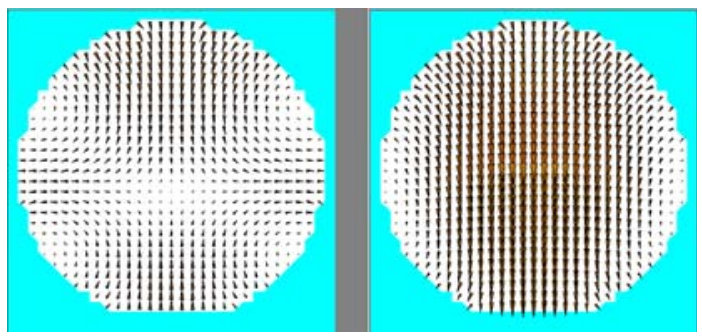

Figure 4: Quadrupole and dipole fields near the collimator

of bunch length. The loss factor is calculated from a wake potential computation. We assume a 14 nano Coulomb gaussian bunch propagating through the round collimator chamber of figure 1. We use MAFIA[3] to calculate the wake potentials and integrate over the bunch charge to determine the loss factor.

Figure 2 shows the loss factor as a function of horizontal and vertical beam offset relative to the collimator. It predicts a strong dependence on vertical position indicating HOM generation will share this feature.

Figure 3 shows the loss factor as a function of bunch length at a fixed position offset. The fit is quadratic which agrees with experimental observations. Figure 4 shows transverse modes excited by the beam near the collimator.

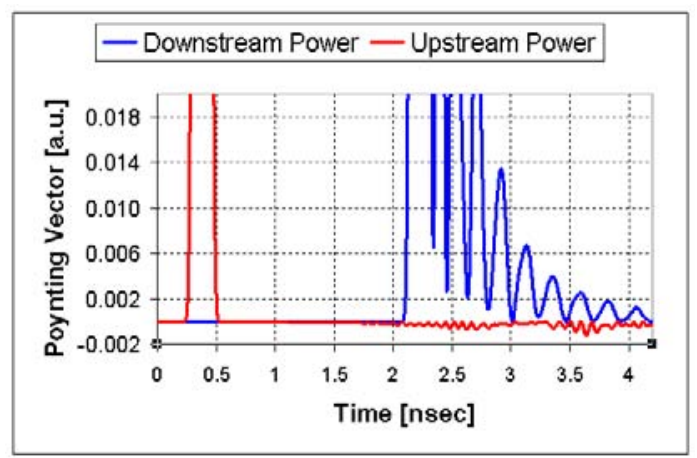

Figure 5: Upstream and downstream HOM propagation

\section{HOM Power Flux}

To investigate HOM propagation it would be useful to calculate the Poynting vector through the collimator structure after the passage of the beam. We use MAFIA to simulate a $1.3 \mathrm{~cm} 14$ nano Coulomb gaussian bunch in our collimator model. We monitor electric and magnetic fields during and after the passage of the beam at two planes upstream and downstream of the collimator. From this data a time dependent power flux can be constructed. Figure 5 shows the total flux of the $z$-component of the Poynting vector $\frac{1}{\mu_{0}} \int(\boldsymbol{E} \times \boldsymbol{B})_{z} d a$ through a vertical plane of circular cross sectional area $a$ at two locations just upstream and downstream of the collimator, separated by a distance of 0.7763 meters. The two large peaks mark the passing of the beam at those location. Here we see a relatively small amount of power propagating backwards from the collimator through an upstream position, while downstream significantly more power propagates forward.

\section{SCATTERING PARAMETER ANALYSIS}

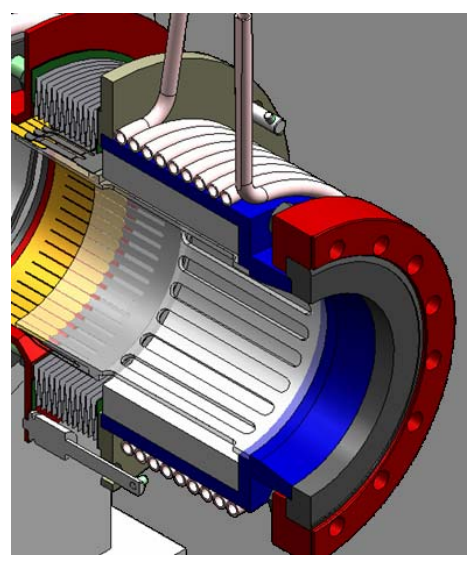

Figure 6: CAD of the straight bellows chamber with water cooled absorber

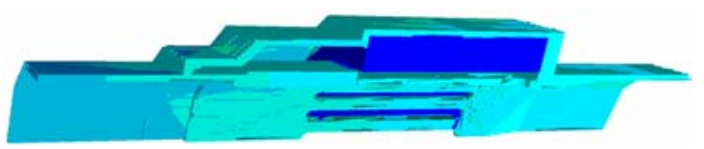

Figure 7: One quarter symmetric mesh for $s$-parameter analysis. Dark blue volume indicates ceramic absorber material.

In order to optimize beam impedance and HOM damping we approach the problem by assuming propagating modes to take the form of transverse monopole, dipole and quadrupole waveguide modes. They are generated by beam fields scattering from some nearby HOM source. We assume the monopole mode to be closely related to the beam field and wish to reduce the impedance to this mode. Alternatively, the dipole and quadrupole modes must be damped since they represent undesirable HOMs. 

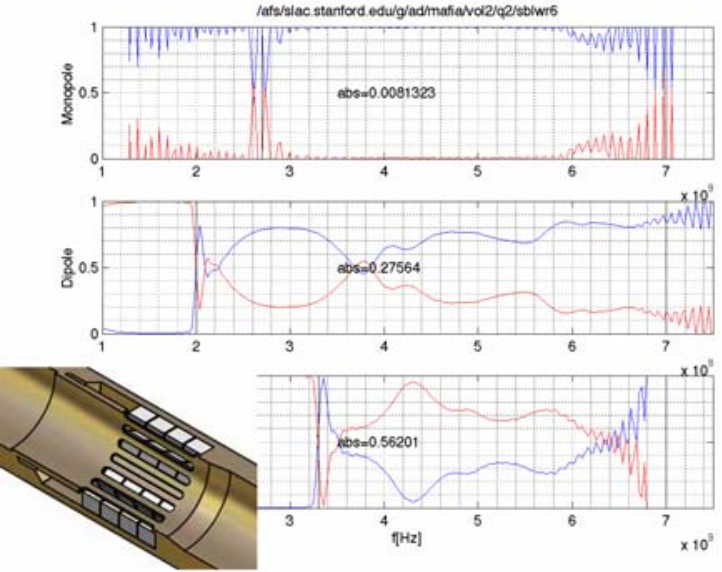

Figure 8: Fractional power loss (blue) and absorption (red) for $6 \mathrm{~mm}$ slots and $12.7 \mathrm{~mm}$ thick absorber
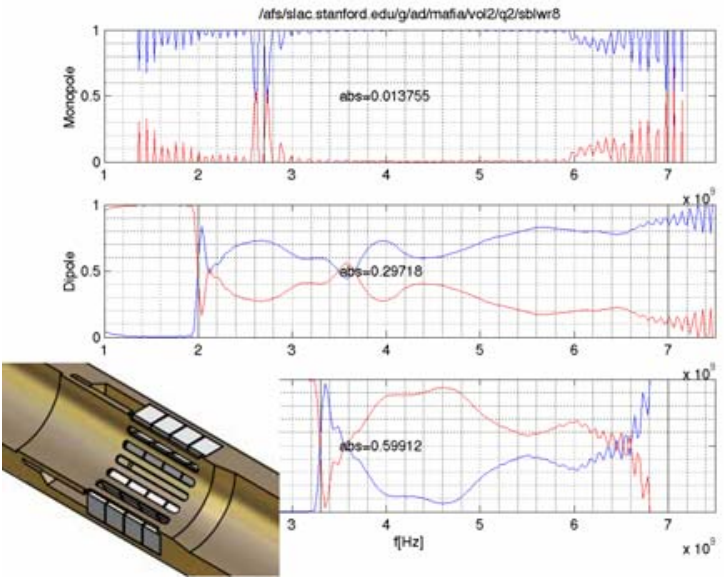

Figure 9: Fractional power loss (blue) and absorption (red) for $6 \mathrm{~mm}$ slots and $16.7 \mathrm{~mm}$ thick absorber

To characterize the behavior of a absorber configuration (geometry and material parameters) $s$-parameter analysis is used. For a given absorber configuration, simulated electromagnetic waves of a particular mode (monpole, dipole or quadrupole) for a range of frequencies propagates through the structure. The wave is matched at the entry and exit ports of the structure. The phase and amplitude of transmitted and reflected waves are monitored at the entry and exit ports of the structure from which the $s$-parameters $s_{11}$ and $s_{21}$ are calculated. Fractional power loss and absorption are given by $s_{11}^{2}+s_{21}^{2}$ and $1-s_{11}^{2}-s_{21}^{2}$ respectively.

Our CAD vacuum chamber model is depicted in figure 7 which roughly simulates the real design in figure 6 . The absorber cavity features an extension which exposes the bellows cavity to the absorber. This has the added effect of damping any modes which might be produced by RF power coupling into the bellows through the bellows fingers. The dark blue material in figure 7 represents a commercially available ceramic with a relative permittivity of
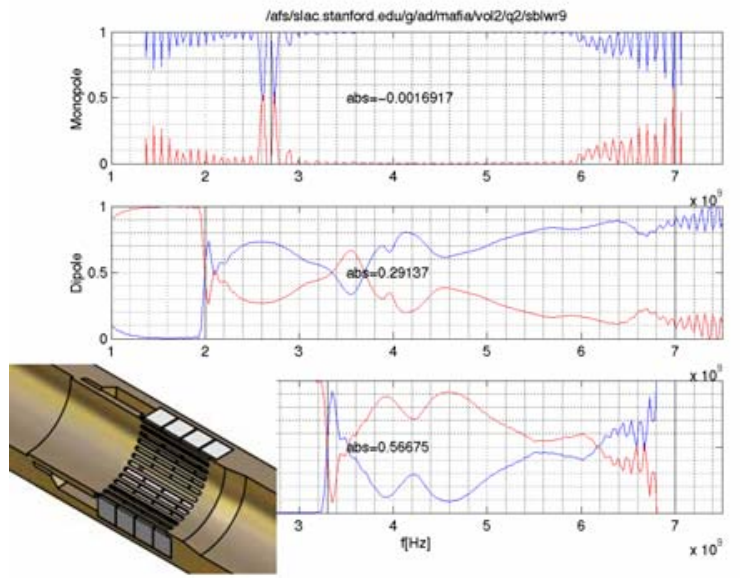

Figure 10: Fractional power loss (blue) and absorption (red) for $3 \mathrm{~mm}$ slots and $16.7 \mathrm{~mm}$ thick absorber

30 and a loss tangent of 0.11 . The upstream and downstream boundaries are assumed to be waveguide ports.

Three configurations are presented representing different geometries. Figure 8 is a plot of fractional power loss and absorption vs frequency for the monopole, dipole and quadrupole waveguide excitation for which the slot width is $6.0 \mathrm{~mm}$ and the absorber thickness is $12.7 \mathrm{~mm}$. Figure 9 is the same plot for the slot width of $6.0 \mathrm{~mm}$ but a thicker absorber of $16.7 \mathrm{~mm}$. Figure 10 is for a smaller slot width of $3.0 \mathrm{~mm}$ also with a thick $16.7 \mathrm{~mm}$ absorber.

The vacuum chamber material (teal colored) has an anular cavity which houses the bellows assembly and a set of ceramic bricks or tiles (dark blue) separated from the beam chamber by a series of longitudinal coupling slots. This offers a conductive path for monopole-like beam fields while exposing the absorbing material to the HOMs in a manner that minimizes impedance the seen by the beam. This is verified by the small monopole absorption. The thickness of the tile is tailored to shift the absorption peak to the desired range of effectiveness.

\section{CONCLUSION}

We present a design for a bellows structure in a LER straight section which incorporates water cooled ceramic absorbers. This device will efficiently damp HOMs produced from nearby fixed vertical and horizontal collimators.

\section{REFERENCES}

[1] A. Novokhatski, et al, "Damping the High Order Modes in the Pumping Chamber of the PEP-II Low Energy Ring", EPAC'04,p. 854.

[2] J. Seeman, "Performance of the PEP-II e+e- Collider at SLAC', PAC'05, May 2005, these proceedings.

[3] The MAFIA collaboration, "User Guide" CST GmbH, Darmstadt, Germany. 